\title{
Desenvolvimento de fluidos aquosos com bentonita para perfuração de poços de petróleo onshore
}

\section{(Development of aqueous fluids with bentonite clay for drilling of onshore oil wells)}

\author{
R. C. A. M. Nascimento ${ }^{1}$ L. V. Amorim ${ }^{2}$, L. N. L. Santana ${ }^{1}$ \\ ${ }^{1}$ Unidade Acadêmica de Engenharia de Materiais, \\ UFCG, Av. Aprígio Veloso 882, Campina Grande, PB 58109-970 \\ ${ }^{2} \mathrm{PRH}-25 \mathrm{ANP}$ \\ nalealves@hotmail.com
}

\begin{abstract}
Resumo
Devido aos grandes desafios encontrados na perfuração de poços, há a necessidade de se desenvolver fluidos com propriedades adequadas e capazes de atender todas as exigências da perfuração, como elevada capacidade de carreamento de sólidos e alta lubricidade, como também fluidos ambientalmente corretos. O grau de impacto dos fluidos de perfuração no meio ambiente depende especialmente do tipo de fluido utilizado, se de base aquosa, oleosa, sintética ou mesmo de base ar. Portanto, é de fundamental importância o desenvolvimento de fluidos com características específicas em cada etapa da perfuração. Sendo assim, este trabalho objetivou o desenvolvimento de fluidos aquosos, com baixo teor de sólidos e propriedades reológicas, de filtração e lubricidade adequadas à perfuração de poços de petróleo onshore. Os fluidos foram formulados utilizando em sua composição bentonita sódica industrializada, aditivos poliméricos e lubrificantes, em diferentes concentrações. O estudo reológico, de filtração e lubricidade mostrou que os fluidos apresentam comportamento pseudoplástico e que os aditivos selecionados desempenham com êxito suas funções de modificadores reológicos, redutores de filtrado e agente lubrificante. Além disto, ficou evidenciada a necessidade de aditivos lubrificantes em fluidos aquosos contendo argila bentonítica e polímeros. A adição de $1 \%$ de lubrificante no fluido é suficiente para adequar a capacidade lubrificante dos fluidos.
\end{abstract}

Palavras-chave: argilas, polímeros, lubrificantes, fluidos de perfuração aquosos.

\begin{abstract}
Due to challenges encountered in drilling wells, there is a need to develop fluids that have properties suitable and able to meet all the requirements of drilling, such as, high capacity for carrying of solid and high lubricity. Besides that, they need to be environmentally friend. The degree of impact of drilling fluids in the environment depends on the type of fluid used, if water or oil or synthetic or air based. It is therefore of fundamental importance to develop fluid with specific characteristics in each stage of drilling. Thus, the aim of this work is to develop aqueous fluids with low content of solids and rheological, filtration and lubricity properties adjusted for drilling of onshore oil wells. The fluids were formulated by using industrialized sodium bentonite, polymeric additives and lubricants, in different concentrations, in its composition. Rheological, filtration and lubricity studies showed that the fluids have pseudoplastic behavior and, the selected additives successively play their functions of rheological modifiers, filtrate reducer and lubricant agent. Moreover, it was evidenced the necessity of lubricant additives in aqueous fluids containing bentonite clay and polymers. The addition of $1 \%$ of lubricant in the fluid is sufficient to adjust the lubricant capacity of fluids.
\end{abstract}

Keywords: clays, polymers, lubricants, aqueous drilling fluids.

\section{INTRODUÇÃO}

Os fluidos de perfuração são definidos geralmente como composições frequentemente líquidas, destinadas a auxiliar o processo de perfuração de poços de petróleo [1]. Esses fluidos são indispensáveis durante as operações de perfuração. O sucesso da perfuração depende do bom desempenho dos fluidos, que por sua vez é determinado de acordo com as funções exercidas pelo fluido, como resfriar e lubrificar a broca de perfuração, reduzir o atrito entre a coluna de perfuração e as paredes do poço, manter a estabilidade do poço, transportar os detritos de perfuração, permitir sua separação na superfície e formar um filme de baixa permeabilidade (reboco) nas paredes do poço [2]. Os fluidos utilizados em cada etapa de perfuração são determinados de acordo com as necessidades do poço. A composição do fluido é selecionada de modo a obter as propriedades ideais, como viscosidades, consistência de gel, controle de filtrado, reboco, inibição de argilas hidratáveis e coeficiente de lubricidade. Nos cenários da 
perfuração de poços, as exigências ambientais estão cada vez mais rigorosas, sendo, portanto, um fator determinante. Com isso, os fluidos à base de água, estão em crescente utilização, visto que fluidos à base de óleo, embora apresentem desempenho superior e sejam mais estáveis a altas pressões e temperaturas, são altamente tóxicos e de custo elevado. Dentre os fluidos aquosos, destacam-se os hidroargilosos e os levemente tratados, recomendados para perfuração de camadas rochosas superficiais, compostas na maioria das vezes de sedimentos inconsolidados [3]. Em perfurações simples e pouco profundas um fluido constituído de água e argila bentonítica é adequado e em situações de difícil perfuração e/ou grandes profundidades é necessário um fluido mais elaborado, com introdução de um ou vários aditivos [4]. O desenvolvimento de um fluido mais elaborado depende das propriedades finais desejadas. Sendo assim, nos fluidos hidroargilosos são utilizadas argilas bentoníticas sódicas com a finalidade de aumentar a viscosidade do meio e formar o reboco, camada de baixa permeabilidade formada na parede do poço para impedir as perdas por filtração. Porém, em baixas concentrações $(2,5 \%)$ essas propriedades não são alcançadas [5]. As propriedades reológicas destes fluidos se tornam adequadas ao serem adicionados aditivos poliméricos. Assim, para promover um aumento da viscosidade, geralmente são utilizados aditivos poliméricos de alta massa molecular. Viscosificantes são utilizados para conferir viscosidade (medida da resistência interna do fluido ao escoamento). Um dos aditivos mais utilizados é carboximetilcelulose (CMC), que pode agir aumentando a viscosidade ou reduzindo o filtrado, e poliacrilamida parcialmente hidrolisada (PHPA), que atua como inibidor e encapsulador de argilas hidratáveis, mas não forma reboco, o que facilita a entrada de sólidos permeáveis na formação [6]. A CMC de alta massa molecular aumenta a viscosidade nos fluidos contendo argila, devido às interações físicas formadas pelas ligações fracas (forças de dispersão), causada pelos ânions carboxilatos presentes na molécula de CMC e as cargas presentes na argila, favorecida pela hidratação da cadeia polimérica e da argila em meio aquoso. O uso da CMC de baixa massa molecular reduz as perdas por filtração e produz rebocos muito finos e capazes de impedir o escoamento do fluido através das formações geológicas que estão sendo perfuradas [7].

A perfuração de poços em profundidades cada vez maiores, bem como poços direcionais e de longa extensão são cada vez mais freqüentes e tem exigido o desenvolvimento de tecnologias sofisticadas e específicas. Um dos principais problemas encontrados principalmente em perfurações com poços direcionais são as forças de friç̧ão entre a coluna de perfuração e as paredes do poço, que podem causar sérios problemas, como o torque excessivo na coluna de perfuração e a diminuição da taxa de penetração. Esses problemas também são freqüentes em poços verticais, independente da profundidade. Alto torque e arraste podem exceder a capacidade dos equipamentos de perfuração e no caso de perfurações direcionais, excederem o limite do afastamento horizontal do poço. Dentre os muitos elementos que afetam o torque e o arraste, o coeficiente de fricção é o fator determinante [8]. Estes problemas podem ser minimizados, ou até mesmo evitados, pelo uso correto de fluidos de perfuração. Para isto, torna-se essencial o uso de fluidos com adequada capacidade de lubrificação, alcançada por meio da aditivação com lubrificantes. Os lubrificantes são substâncias que colocadas entre duas superfícies formam uma película protetora que tem como função principal reduzir o atrito, o desgaste, auxiliar no controle da temperatura, proporcionando a limpeza do equipamento, protegendo contra a corrosão decorrente dos processos de oxidação, podendo também, ser agente de transmissão de força e movimento [9]. Fluidos lubrificantes podem proporcionar uma série de benefícios nas operações de perfuração, como aumento da vida útil da broca, aumento da taxa de perfuração, redução do torque, aumento da estabilidade do poço que auxilia no controle da temperatura [9]. Os fluidos de base oleosa são considerados como fluidos que possuem excelente lubricidade, com coeficiente de lubricidade (CL) em torno de 0,15 [2]. Por outro lado, os fluidos à base de água apresentam valores de CL entre 0,35 e 0,50 e os fluidos à base de água com $4,3 \%$ de bentonita, valores de 0,44 .

Este trabalho teve como objetivo desenvolver fluidos aquosos, com baixo teor de sólidos e propriedades reológicas, de filtração e lubricidade adequadas à perfuração de poços de petróleo onshore.

\section{MATÉRIAIS E MÉTODOS}

$\mathrm{Na}$ Tabela I estão apresentadas as composições dos fluidos que contém em sua composição apenas a argila.

Tabela I - Fluidos de perfuração preparados com argila. [Table I - Drilling fluids prepared with clay.]

\begin{tabular}{ccc}
\hline Fluidos & Água $(\mathrm{mL})$ & Argila $(\%)$ \\
\hline F1 & 500 & 2,5 \\
F2 & 500 & 4,86 \\
\hline
\end{tabular}

Foi estudada uma amostra de argila bentonítica sódica industrializada da Paraíba, conhecida comercialmente por Brasgel PA, nas concentrações de 2,5 e 4,86\% em massa de argila. Esta última concentração foi utilizada com o objetivo de qualificar a argila de acordo com as especificações da Petrobrás [10] para uso em fluidos de perfuração à base de água doce. A argila foi fornecida pela empresa Bentonit União Nordeste Ltda, Campina Grande, PB.

Para adequar as propriedades reológicas e de filtração dos fluidos, foram estudados dois aditivos poliméricos, denominados neste trabalho P1 e P2, nas concentrações de 0,12 e $0,16 \%$ e uma combinação ternária de aditivos, denominada P3, nas concentrações de 0,08 e $0,12 \%$. $\mathrm{O}$ aditivo polimérico $\mathrm{P} 1$ atua como viscosificante, o polímero P2, como redutor de filtrado e a combinação P3, é uma mistura de polímeros que atua como viscosificante, 
Tabela III - Fluidos de perfuração preparados com argila, polímeros e lubrificantes. [Table III - Drilling fluids prepared with clay, polymers and lubricants.]

\begin{tabular}{cccccccccc}
\hline Fluidos & $\begin{array}{c}\text { Água } \\
(\mathrm{mL})\end{array}$ & $\begin{array}{c}\text { Argila } \\
(\%)\end{array}$ & $\begin{array}{c}\text { P1 } \\
(\%)\end{array}$ & $\begin{array}{c}\text { P2 } \\
(\%)\end{array}$ & $\begin{array}{c}\text { P3 } \\
(\%)\end{array}$ & $\begin{array}{c}\text { Lub1 } \\
(\%)\end{array}$ & $\begin{array}{c}\text { Lub2 } \\
(\%)\end{array}$ & $\begin{array}{c}\text { Lub3 } \\
(\%)\end{array}$ & $\begin{array}{c}\text { Lub4 } \\
(\%)\end{array}$ \\
\hline F9 & 500 & 2,5 & 0,16 & --- & --- & 1,0 & --- & --- & --- \\
F10 & 500 & 2,5 & 0,16 & --- & --- & 2,0 & --- & --- & -- \\
F11 & 500 & 2,5 & 0,16 & --- & --- & --- & 1,0 & --- & --- \\
F12 & 500 & 2,5 & 0,16 & --- & --- & --- & 2,0 & --- & --- \\
F13 & 500 & 2,5 & 0,16 & --- & --- & --- & --- & 1,0 & --- \\
F14 & 500 & 2,5 & 0,16 & --- & --- & --- & --- & 2,0 & --- \\
F15 & 500 & 2,5 & 0,16 & --- & --- & --- & --- & --- & 1,0 \\
F16 & 500 & 2,5 & 0,16 & --- & --- & --- & --- & --- & 2,0 \\
F17 & 500 & 2,5 & --- & 0,16 & --- & 1,0 & --- & --- & --- \\
F18 & 500 & 2,5 & --- & 0,16 & --- & 2,0 & --- & --- & --- \\
F19 & 500 & 2,5 & --- & 0,16 & --- & --- & 1,0 & --- & --- \\
F20 & 500 & 2,5 & --- & 0,16 & --- & --- & 2,0 & --- & -- \\
F21 & 500 & 2,5 & --- & 0,16 & --- & --- & --- & 1,0 & --- \\
F22 & 500 & 2,5 & --- & 0,16 & --- & --- & --- & 2,0 & --- \\
F23 & 500 & 2,5 & --- & 0,16 & --- & -- & --- & --- & 1,0 \\
F24 & 500 & 2,5 & --- & 0,16 & --- & --- & --- & --- & 2,0 \\
F25 & 500 & 2,5 & --- & --- & 0,16 & 1,0 & --- & --- & --- \\
F26 & 500 & 2,5 & --- & --- & 0,16 & 2,0 & --- & --- & --- \\
F27 & 500 & 2,5 & --- & --- & 0,16 & --- & 1,0 & --- & --- \\
F28 & 500 & 2,5 & --- & --- & 0,16 & --- & 2,0 & --- & -- \\
F29 & 500 & 2,5 & --- & --- & 0,16 & --- & --- & 1,0 & --- \\
F30 & 500 & 2,5 & --- & --- & 0,16 & --- & --- & 2,0 & --- \\
F31 & 500 & 2,5 & --- & --- & 0,16 & --- & --- & --- & 1,0 \\
F32 & 500 & 2,5 & --- & --- & 0,16 & --- & --- & --- & 2,0 \\
\hline
\end{tabular}

Tabela II - Fluidos de perfuração preparados com argila e polímeros.

[Table II - Drilling fluids prepared with clay and polymer.]

\begin{tabular}{cccccc}
\hline Fluidos & $\begin{array}{c}\text { Água } \\
(\mathrm{mL})\end{array}$ & $\begin{array}{c}\text { Argila } \\
(\%)\end{array}$ & $\begin{array}{c}\text { P1 } \\
(\%)\end{array}$ & $\begin{array}{c}\text { P2 } \\
(\%)\end{array}$ & $\begin{array}{c}\text { P3 } \\
(\%)\end{array}$ \\
\hline F3 & 500 & 2,5 & 0,12 & --- & --- \\
F4 & 500 & 2,5 & 0,16 & --- & --- \\
F5 & 500 & 2,5 & --- & 0,12 & --- \\
F6 & 500 & 2,5 & --- & 0,16 & --- \\
F7 & 500 & 2,5 & --- & --- & 0,08 \\
F8 & 500 & 2,5 & --- & --- & 0,12 \\
\hline
\end{tabular}

redutor de filtrado e encapsulador/estabilizador de argila. As amostras dos polímeros foram fornecidas pela empresa System Mud Ind. Com. Ltda., Itajaí, SC.

$\mathrm{Na}$ Tabela II estão apresentadas as composições dos fluidos que contém em sua composição a argila e aditivos poliméricos.

Com o objetivo de melhorar a lubricidade dos fluidos, foram utilizados quatro tipos de lubrificantes, denominados Lub1, Lub2, Lub3 e Lub4, nas concentrações de 1,0 e 2,0\%. As amostras dos quatro lubrificantes foram fornecidas pela empresa System Mud Ind. Com. Ltda.. Na Tabela III estão apresentadas as composições dos fluidos que contém em sua composição a argila, aditivos poliméricos e lubrificantes.

\section{METODOLOGIA}

Preparação dos fluidos de perfuração: a argila foi misturada manualmente com o polímero em pó e, em seguida, essa mistura foi adicionada a $500 \mathrm{~mL}$ de água deionizada sob agitação a $17.000 \mathrm{rpm}$ durante $20 \mathrm{~min}$ em um agitador mecânico Hamilton Beach 936. A seguir o fluido permaneceu em repouso durante $24 \mathrm{~h}$ em recipiente fechado e, após esse período, foi adicionado o lubrificante sob agitação constante no mesmo agitador mecânico a 17.000 rpm durante $1 \mathrm{~min}$. Os fluidos de perfuração à base de água foram preparados com base na norma N-2605 [11].

Estudo reológico dos fluidos de perfuração: Após repouso de $24 \mathrm{~h}$, foi realizado o estudo reológico dos fluidos de perfuração. O fluido foi agitado durante 5 min no mesmo agitador mecânico a 17.000 rpm. Após a agitação, o fluido foi transferido para o recipiente do viscosímetro Fann 35A. Seis valores de torque foram lidos com taxas de cisalhamento variando de 5,1 a $1022 \mathrm{~s}^{-1}$. O viscosímetro foi acionado a $600 \mathrm{rpm}$ durante 2 min e efetuada a leitura. Logo após, a velocidade foi mudada para $300 \mathrm{rpm}$, efetuando a leitura após $15 \mathrm{~s}$. Novamente, mudou-se a velocidade para $200 \mathrm{rpm}$ e esperou-se estabilizar para efetuar a leitura. O 
mesmo procedimento foi utilizado para as velocidades de $100 \mathrm{rpm}, 6 \mathrm{rpm}$ e $3 \mathrm{rpm}$. Para obtenção da força gel inicial, o fluido durante $15 \mathrm{~s}$ foi mantido no viscosímetro Fann a 600 rpm e, em seguida, permaneceu em repouso durante $10 \mathrm{~s}$. Logo após, colocou-se na velocidade de $3 \mathrm{rpm}$, efetuandose a leitura. A seguir, para a obtenção da força gel final, o fluido foi deixado em repouso durante $10 \mathrm{~min}$ e, logo após, efetuada a leitura na velocidade de $3 \mathrm{rpm}$. Com os dados das leituras obtidas no viscosímetro, calculou-se a viscosidade aparente (VA), a viscosidade plástica (VP) e o limite de escoamento (LE) segundo a norma N-2605 [11], utilizando as equações abaixo.

Viscosidade aparente (VA):

$$
\mathrm{VA}=\frac{\mathrm{L}_{600}}{2}(\mathrm{cP})
$$

Viscosidade plástica (VP):

$$
\mathrm{VP}=\mathrm{L}_{600}-\mathrm{L}_{300}(\mathrm{cP})
$$

Limite de escoamento (LE):

$$
\mathrm{LE}=\mathrm{L}_{300}-\mathrm{VP}\left(\mathrm{N} / \mathrm{m}^{2}\right)
$$

O coeficiente de lubricidade dos fluidos foi determinado em lubricímetro Ofite (EP- Lubricity Tester). Utilizou-se a metodologia sugerida pelo fabricante do equipamento, que consiste em agitar o fluido por $5 \mathrm{~min}$ no agitador mecânico a $17.000 \mathrm{rpm}$. Em seguida, o fluido foi transferido para o recipiente do equipamento, com torque inicial zero e uma velocidade de $60 \mathrm{rpm}$; aplicou-se lentamente uma força de $150 \mathrm{in} / \mathrm{lb}$ (equivalente a uma pressão de 34.500 a $69.000 \mathrm{kPa}$ ) durante $5 \mathrm{~min}$, efetuando-se a leitura do torque exercido pelo fluido. Com a leitura obtida pelo torque da água, calculouse o fator de correção (FC), de acordo com a equação D e o coeficiente de lubricidade de acordo com a equação $\mathrm{E}$.

Fator de correlação:

$$
\mathrm{FC}=\frac{34,0}{\text { Leitura }_{\text {agua }}}
$$

Coeficiente de lubricidade:

$$
\mathrm{CL}=\frac{\mathrm{FC}-\text { Leitura }_{\text {obtida }}}{100}
$$

Para a determinação do volume de filtrado, os fluidos foram agitados durante $1 \mathrm{~min}$, no agitador mecânico a 17.000 rpm. Em seguida, o fluido foi transferido para o recipiente do filtro-prensa API, com aplicação de $7,0 \mathrm{kgf} / \mathrm{cm}^{2}$ (100 psi). Após $30 \mathrm{~min}$, o filtrado foi lido e obteve-se a medida do volume do filtrado, expresso em $\mathrm{mL}$.

Para determinar a espessura do reboco foi utilizada a metodologia desenvolvida LABDES (Laboratório de Referência de Dessalinização) [12] baseada na norma API 13B-1 [13]. Consiste em coletar o papel de filtro com o reboco após a realização do ensaio para determinação do volume do filtrado, lavar o papel de filtro três vezes a uma vazão de aproximadamente $110 \mathrm{~L} / \mathrm{h}$ com o auxílio de um recipiente de nível constante com vazão regulável, colocar papel de filtro com o reboco entre duas lâminas de vidro e aplicar aproximadamente $277,6 \mathrm{~N} / \mathrm{m}^{2}$ por 2 min e medir a espessura do reboco com o auxílio de um extensômetro. Foram feitas cinco medidas das espessuras das lâminas de vidro e do papel com o reboco em pontos distintos e obtida a média aritmética. Todos os ensaios foram realizados em duplicata. Quando um dos valores obtidos diferia da média de $10 \%$, esse era automaticamente eliminado e o ensaio repetido.

A Fig. 1 apresenta as curvas de viscosidade dos fluidos hidroargilosos (F1 e F2). Os fluidos F1 e F2 apresentaram comportamento de fluidos binghamianos, independente da concentração de argila estudada.

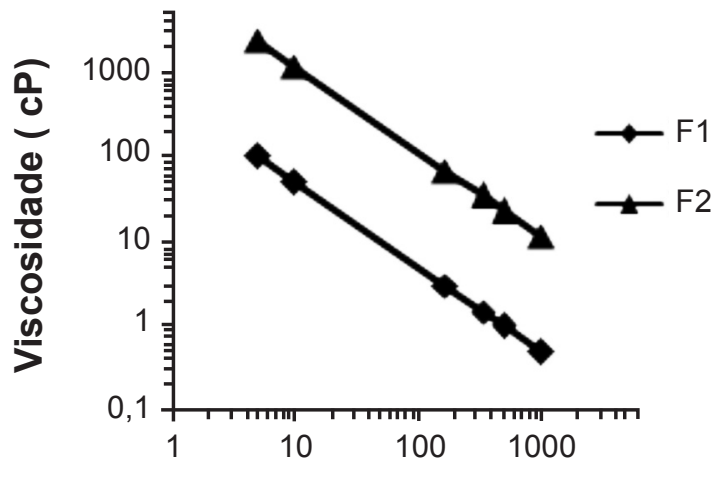

Taxa de cisalhamento (1/s)

Figura 1: Curvas de viscosidade dos fluidos preparados com argila. [Figure 1: Curves of viscosity of fluids prepared with clay.]

Na Tabela IV estão apresentados os valores das propriedades reológicas (viscosidade aparente VA, viscosidade

Tabela IV - Propriedades reológicas e de filtração dos fluidos de perfuração F1e F2.

[Table IV - Rheological properties and filtration of drilling fluids F1e F2.]

\begin{tabular}{cccccccc}
\hline Fluidos & $\mathrm{VA}(\mathrm{cP})$ & $\mathrm{VP}(\mathrm{cP})$ & $\mathrm{LE}\left(\mathrm{N} / \mathrm{m}^{2}\right)$ & $\mathrm{FG}\left(\mathrm{N} / \mathrm{m}^{2}\right)$ & $\mathrm{VF}(\mathrm{mL})$ & $\mathrm{ER}(\mathrm{mm})$ & $\mathrm{CL}$ \\
\hline $\mathrm{F} 1$ & 4,5 & 3,0 & 3,0 & 3,0 & 26,0 & 1,054 & 0,38 \\
F2 & 20,0 & 4,0 & 32,0 & 6,0 & 17,0 & 2,157 & 0,4 \\
Especificações & & & & & & & \\
Petrobrás [11] & $\geq 15,0$ & $\geq 4,0$ & $\leq 1,5 \mathrm{xVP}$ & $\mathrm{NE}$ & $\leq 18,0$ & $\mathrm{NE}$ & $\mathrm{NE}$ \\
\hline
\end{tabular}


plástica VP, limite de escoamento LE, força gel FG) e de filtração (volume do filtrado VF e espessura do reboco ER) e lubricidade CL dos fluidos de perfuração F1e F2.

Observou-se um aumento nos valores de VA, VP, LE e FG com o aumento da concentração de argila. $\mathrm{O}$ aumento nos valores de VA decorre da maior intensidade das interações eletrostáticas e de massa entre as partículas sólidas, que se tornam predominantes com o aumento da concentração de argila [14]. Mesmo em baixas concentrações de argila, observou-se que os fluidos apresentaram ainda a formação de gel, isto acontece porque as partículas de argila presentes nos fluidos de perfuração são anisométricas e podem formar uma estrutura de gel mesmo em baixas concentrações de sólidos [2]. Os resultados de VF reduziram à medida que aumentou a concentração de argila. Como mencionado anteriormente, a maior concentração de argila no meio proporciona uma maior intensidade eletrostática e de massa entre as partículas. Essas interações, por sua vez, promovem a formação de reticulados mais ou menos rígidos, que retêm as moléculas de água diminuindo, assim, a quantidade de água livre no sistema, reduzindo, conseqüentemente, VF. Os resultados obtidos de ER mostraram que o aumento na concentração de argila proporcionou rebocos mais espessos, como esperado. Portanto, observou-se que os fluidos preparados com 4,86\% de argila apresentaram valores de VA, VP e VF de acordo com as especificações da Petrobrás [10], qualificando a mesma para aplicação em fluidos à base de água doce. Podese observar que CL não apresenta variações significativas com o aumento da concentração de argila bentonítica utilizada no preparo do fluido de perfuração. Esses valores são próximos ao CL $(0,44)$ de fluidos preparados com $4,3 \%$ de bentonita [2]. De acordo com a literatura, fluidos com lubricidade adequada devem apresentar valores de CL da ordem de 0,1, valor comumente encontrado em fluidos de perfuração base óleo ou em fluidos aditivados com agentes lubrificantes. Desta forma, fica evidente que as argilas bentoníticas não contribuem para a melhoria da lubricidade de fluidos aquosos, e que CL independe da concentração de bentonita no fluido. Para melhorar a lubricidade de fluidos hidroargilosos, há a necessidade da adição de aditivos lubrificantes. Como foi comprovada a qualidade da argila de acordo com as especificações [10] da Petrobrás, a mesma foi utilizada para o preparo dos demais fluidos, contudo optou-se por utilizar a concentração de $2,5 \%$ de argila, visando atingir um dos objetivos deste trabalho, que é o desenvolvimento de fluidos com baixo teor de sólidos.

A adição dos polímeros ao fluido ocasionou mudança do comportamento reológico, passando de comportamento de fluidos binghamianos para comportamento de fluidos pseudoplásticos com limite de escoamento. Essa mudança no comportamento reológico ocorre porque os aditivos poliméricos apresentam comportamento pseudoplástico [15]. A Fig. 2 apresenta as curvas de viscosidade dos fluidos preparados com argila após aditivação com polímeros. Observou-se que a viscosidade diminui com o aumento da taxa de cisalhamento, caracterizando comportamento pseudoplástico.

$\mathrm{Na}$ Tabela $\mathrm{V}$ estão apresentados os resultados das propriedades reológicas e de filtração e lubricidade dos fluidos preparados com argila após aditivação polimérica.

De maneira geral, os fluidos aditivados com polímeros apresentaram valores de VA, VP e LE superiores e satisfatórios quando comparados com o fluido sem aditivação (F2), o que confirma a ação dos polímeros, que além de agir como viscosificantes atuam também como redutores de filtrado. Nos fluidos aditivados com P3 (F7 e F8), os valores de VA e VP são bem mais elevados que os apresentados pelos fluidos aditivados com P1 (F3 e F4) e P2 (F5 e F6). O comportamento desses fluidos está de acordo com o esperado, pois nos fluidos F7 e F8, há a presença de $\mathrm{CMC}$ de alta viscosidade (CMC AV), que é um polímero de cadeia muito longa (de elevada massa molecular e da poliacrilamida parcialmente hidrolisada (PHPA), que pode ocasionar um estado floculado, pois várias partículas de argila são adsorvidas na mesma cadeia polimérica gerando o fenômeno de encapsulamento e/ou formação de flocos dificultando o escoamento do fluido e resultando em uma maior viscosidade [16]. Os fluidos F5 e F6 apresentaram melhores resultados de VF, pois o polímero P2, polímero de baixa viscosidade, devido à sua baixa massa molar, atua
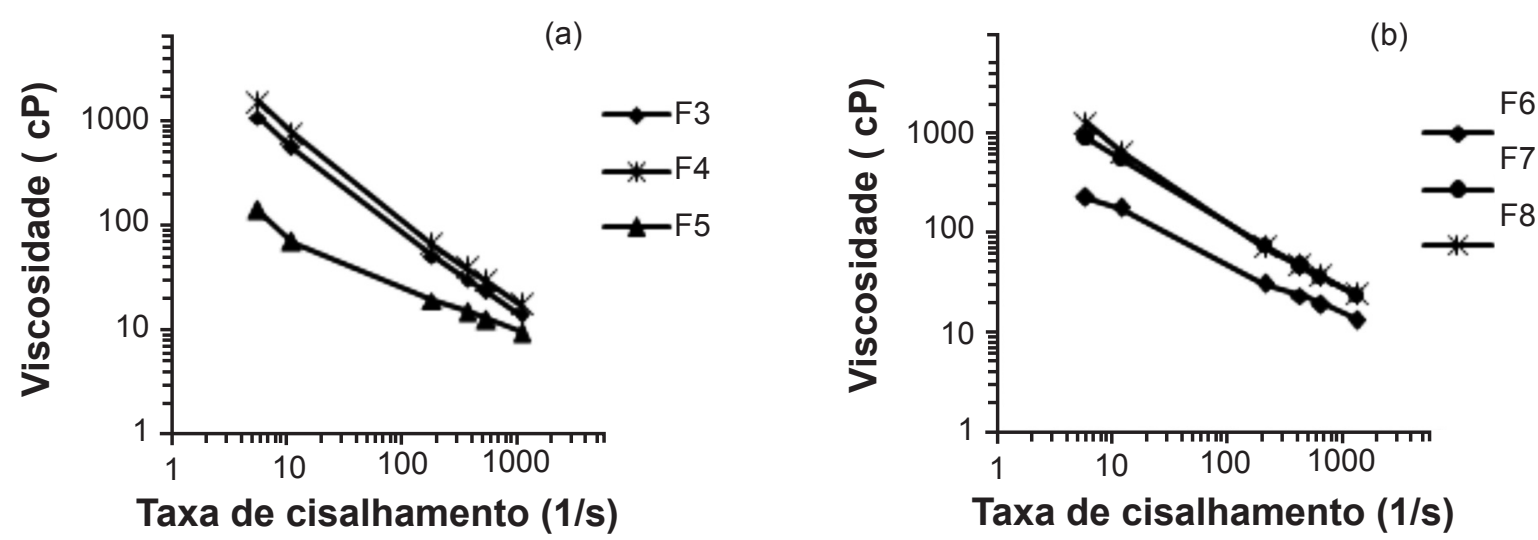

Figura 2: Curvas de viscosidade dos fluidos de perfuração preparados com argila e polímeros.

[Figure 2: Curves of viscosity of drilling fluids prepared with clay and polymer.] 
Tabela V - Propriedades reológicas e de filtração dos fluidos de perfuração preparados com a argila e aditivos poliméricos.

[Table V-Rheological properties and filtration of drilling fluids prepared with clay and polymeric additives.]

\begin{tabular}{cccccccc}
\hline Fluidos & $\begin{array}{c}\mathrm{VA} \\
(\mathrm{cP})\end{array}$ & $\begin{array}{c}\mathrm{VP} \\
(\mathrm{cP})\end{array}$ & $\mathrm{LE}\left(\mathrm{N} / \mathrm{m}^{2}\right)$ & $\begin{array}{c}\mathrm{FG}(\mathrm{N} / \\
\left.\mathrm{m}^{2}\right)\end{array}$ & $\begin{array}{c}\mathrm{VF} \\
(\mathrm{mL})\end{array}$ & $\begin{array}{c}\mathrm{ER} \\
(\mathrm{mm})\end{array}$ & $\mathrm{CL}$ \\
\hline F2 & 4,5 & 3,0 & 3,0 & 3,0 & 26,0 & 1,054 & 0,38 \\
F3 & 15,3 & 5,0 & 20,5 & 3,0 & 17,2 & 1,239 & 0,38 \\
F4 & 19,3 & 6,5 & 25,5 & 4,0 & 14,0 & 1,172 & 0,36 \\
F5 & 10,5 & 7,0 & 7,0 & 8,0 & 15,6 & 1,399 & 0,39 \\
F6 & 15,0 & 8,5 & 13,0 & 11,0 & 12,0 & 1,470 & 0,39 \\
F7 & 26,0 & 12,0 & 28,0 & 1,0 & 16,4 & 1,638 & 0,31 \\
F8 & 27,0 & 13,0 & 28,0 & 6,5 & 16,0 & 1,273 & 0,31 \\
Especificações & & & & & & & \\
Petrobrás [11] & $\geq 15,0$ & $\geq 4,0$ & $\leq 1,5 \mathrm{xVP}$ & $\mathrm{NE}$ & $\leq 18,0$ & $\mathrm{NE}$ & $\mathrm{NE}$ \\
\hline
\end{tabular}

de forma mais eficiente na redução de VF. Os altos valores de LE dos fluidos após a adição de polímeros devem-se à expansão da cadeia polimérica durante a sua hidratação que, por sua vez, adquirem uma configuração alongada e elevam a viscosidade, visto que o LE depende da VP que é uma medida de fricção resultante do choque das partículas entre si [6]. Os coeficientes de lubricidade dos fluidos estudados tiveram pouca variação, permanecendo quase que constantes, variando entre 0,31 para os fluidos F7 e F8 a 0,39 para os fluidos F5 e F6. Como já mencionado, fluidos base óleo apresentam $C L$ de 0,15 , enquanto os fluidos à base de água, valores entre 0,35 e 0,50 e fluidos base água com $4,3 \%$ de bentonita, 0,44 [2]. Embora tenha sido observada uma discreta redução do CL para alguns fluidos, concluiu-se que esses aditivos poliméricos não auxiliam na lubrificação dos fluidos de perfuração. A partir da análise dos resultados apresentados, foram selecionados os fluidos F4, F6 e F7 para aditivação com agentes lubrificantes, com a finalidade de se obter fluidos com baixo teor de sólidos e propriedades reológicas, de filtração e coeficiente de lubricidade adequados.

Assim como nos fluidos preparados com argila e aditivados com polímeros, os fluidos estudados nesta etapa, que além da argila e dos polímeros contém aditivos lubrificantes, apresentaram comportamento de fluidos pseudoplásticos. Como todos os fluidos apresentaram comportamentos semelhantes, foram selecionados os fluidos F9, F11, F13, F15, F17, F19, F21, F23, F25, F27, F29 e F31, para ilustrar as curvas de viscosidade (Fig. 3).

$\mathrm{Na}$ Tabela VI estão apresentados os resultados das propriedades reológicas, de filtração e lubricidade dos fluidos preparados com argila após aditivação polimérica e lubrificantes.

Com os resultados apresentados na Tabela VI, observouse que os valores de VA para os fluidos F9 a F16 e F25 a F32 reduziu com a adição do lubrificante, enquanto que para a maioria dos fluidos F17 a F24 a viscosidade aumentou com a adição do lubrificante. Os fluidos F10 e F17 não apresentaram VA de acordo com o valor especificado [10]. A pequena redução dos valores de VA deve-se, provavelmente, à interação entre o lubrificante e as partículas de argila; o lubrificante quando adicionado ao fluido contendo argila age como dispersante, blindando as cargas presentes nas superfícies das partículas de argila e, portanto, tem-se um efeito afinante, reduzindo a viscosidade. Os maiores resultados de VA foram obtidos para os fluidos F25 a F32, isto porque, os polímeros presentes na composição P3 são característicos de polímeros de cadeia longa. Os valores de VP praticamente não variaram com a adição do lubrificante, permanecendo todos, de acordo com os padrões especificados pela Petrobrás [10] para qualificação de bentonitas ativadas. O LE variou de $11,5 \mathrm{~N} / \mathrm{m}^{2}$ para os fluidos F12, F19 e F21 a $27,0 \mathrm{~N} / \mathrm{m}^{2}$ para o fluido $\mathrm{F} 31$. A FG variou dependendo do polímero utilizado e do tipo e teor de lubrificante; observouse um valor máximo de $27,0 \mathrm{~N} / \mathrm{m}^{2}$ para os fluidos $\mathrm{F} 21$. O grau de tixotropia do fluido é obtido pela FG que é um parâmetro de natureza reológica que indica o grau de gelificação devido às interações eletrostáticas entre as partículas dispersas [3]. A tixotropia é causada pela presença de partículas carregadas eletricamente que se encadeiam umas às outras, formando uma matriz rígida. Ao adicionar o lubrificante, houve uma redução do VF, o que garantiu melhor comportamento para os fluidos. Os fluidos F17 a F24 apresentaram os menores valores de $\mathrm{VF}$, próximos a $12,0 \mathrm{~mL}$; esse comportamento é explicado porque o polímero (P2) presente na composição destes fluidos, é um polímero de cadeia curta, e assim apresenta atuação mais eficiente na redução de filtrado. O fluido deve apresentar VF adequado, pois a perda excessiva de água para a formação geológica pode gerar diversos problemas, a exemplo do desmoronamento das formações e aprisionamento de brocas. A ER apresentou pequenas variações e esta, por sua vez, está diretamente relacionada com o VF, isto porque um fluido com uma maior quantidade de água presente ao ser submetido a uma determinada pressão resultará em um maior $\mathrm{VF}$, conseqüentemente, o reboco obtido apresentará maior espessura. Para se ter um bom controle durante a perfuração essa relação é bastante importante, pois o excesso de reboco na parede do poço pode causar aprisionamento da coluna de perfuração. Ainda de acordo com a Tabela VI, pode-se observar que os fluidos 
sem a presença do lubrificante (F4, F6 e F7) apresentaram CL próximos de 0,4 e após a sua adição, todos os fluidos apresentaram valores entre 0,08 e 0,10 , inferiores a 0,15 , característicos de fluidos base óleo. Os fluidos com menor coeficiente de lubricidade foram F17 e F18. Observou-se que aumento da concentração do lubrificante tem pouca influência no coeficiente de lubricidade dos fluidos, sendo, portanto, o teor de $1,0 \%$ suficiente para garantir ao fluido adequada capacidade de lubrificação. O desempenho relativo dos lubrificantes estão correlacionados inversamente com o teor de sólidos no fluido e com a atividade de superfície de outros componentes do fluido (quanto maior a quantidade de sólidos no fluido, menor é o desempenho de um lubrificante) [16]. É importante ressaltar que tradicionalmente empresas multinacionais de fluidos utilizam uma concentração de $3,0 \%$ de lubrificante em fluidos base água. Foram reportados valores de CL entre 0,102 (3,0\% de lubrificante) e 0,299 (1,0\% de lubrificante) para fluidos à base de água salgada,
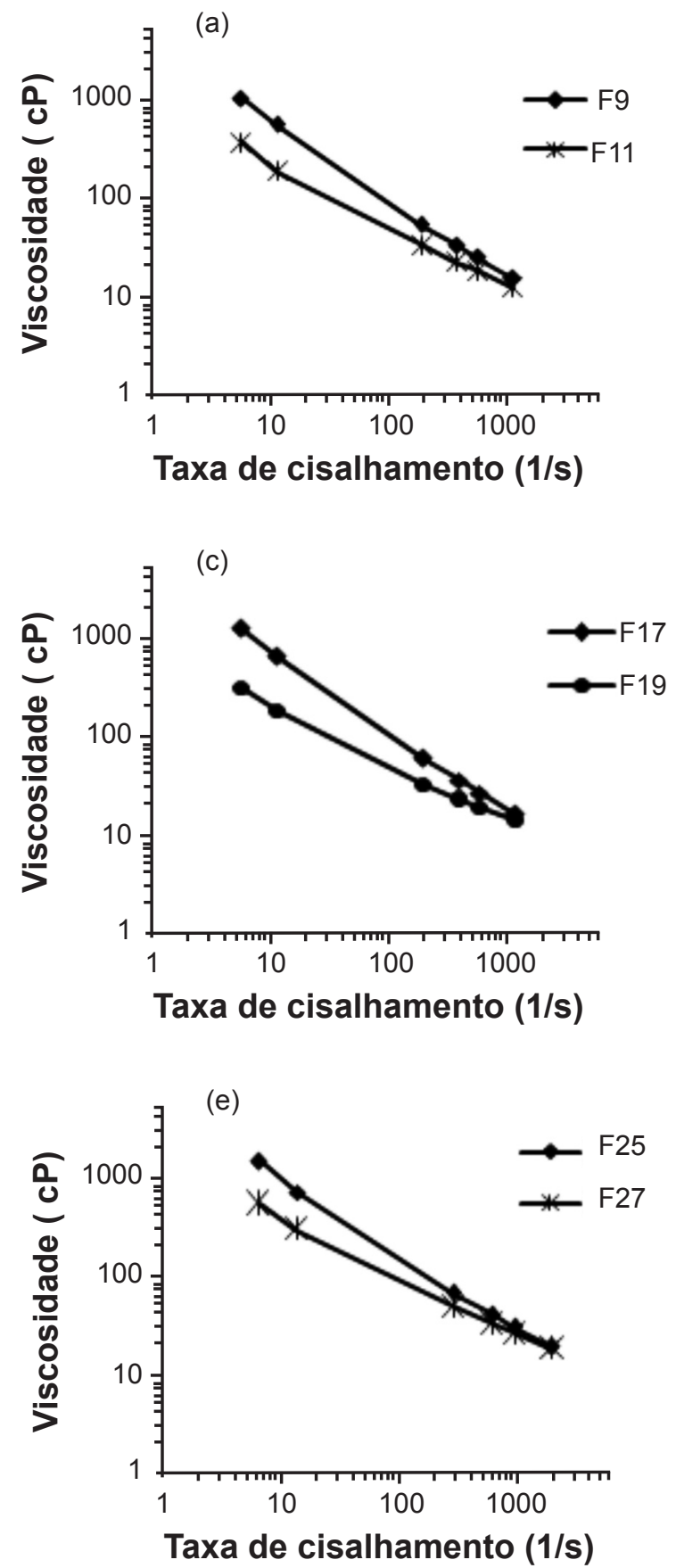
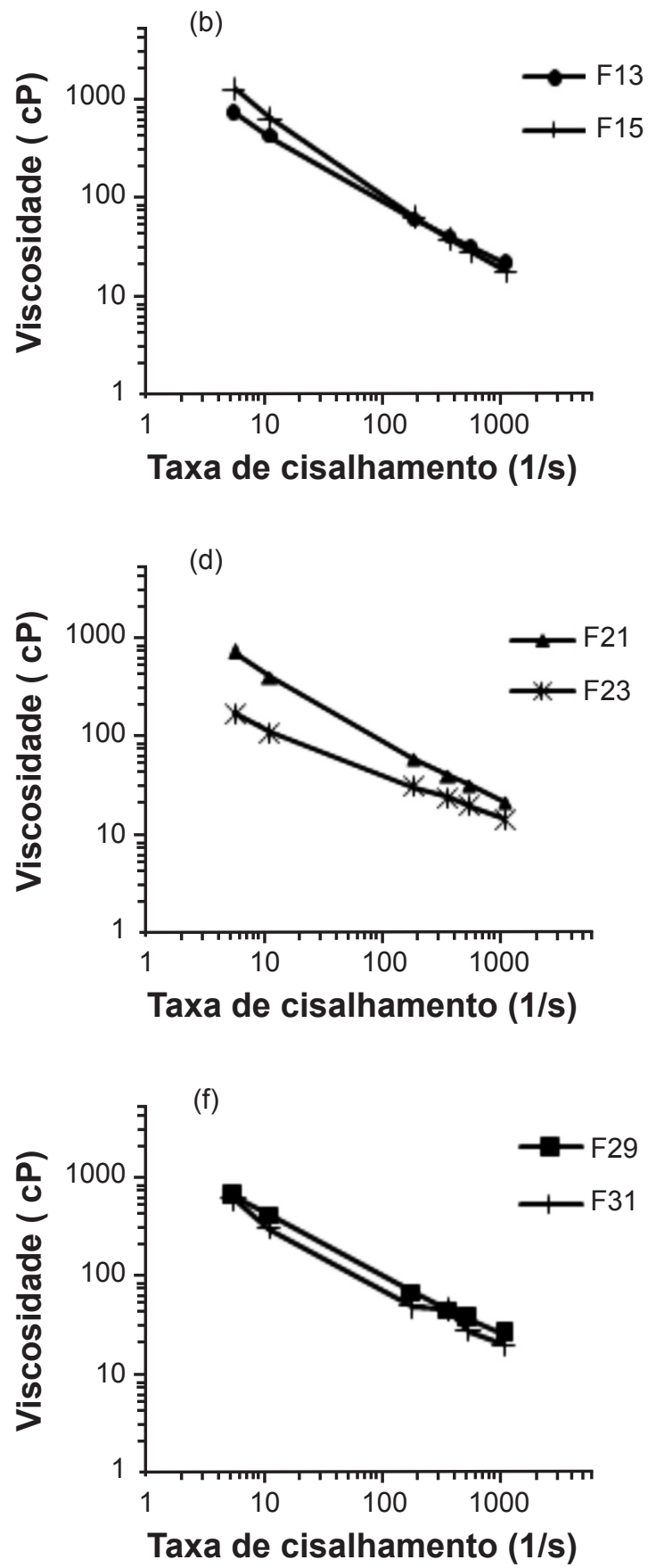

Figura 3: Curvas de viscosidade dos fluidos de perfuração (a) e (b) preparados com argila, P1 e lubrificantes, (c) e (d) preparados com argila, P2 e lubrificantes, (e) e (f) preparados com argila, P3 e lubrificantes.

[Figure 3: Viscosity curves of the drilling fluids (a) e (b) prepared with clay, P1 and lubricants, (c) e (d) prepared with clay, P2 and lubricants, (e) e (f) prepared with clay, P3 and lubricants.] 
Tabela VI - Propriedades reológicas e de filtração dos fluidos de perfuração preparados com a argila, aditivos poliméricos e lubrificantes.

[Table VI - Rheological properties and filtration of drilling fluids prepared with the clay, polymer additives and lubricants.]

\begin{tabular}{cccccccc}
\hline Fluidos & $\begin{array}{c}\text { VA } \\
(\mathrm{cP})\end{array}$ & $\begin{array}{c}\mathrm{VP} \\
(\mathrm{cP})\end{array}$ & $\begin{array}{c}\mathrm{LE} \\
\left(\mathrm{N} / \mathrm{m}^{2}\right)\end{array}$ & $\begin{array}{c}\mathrm{FG} \\
\left(\mathrm{N} / \mathrm{m}^{2}\right)\end{array}$ & $\begin{array}{c}\mathrm{VF} \\
(\mathrm{mL})\end{array}$ & $\begin{array}{c}\mathrm{ER} \\
(\mathrm{mm})\end{array}$ & $\mathrm{CL}$ \\
\hline F4 & 19,3 & 6,5 & 25,5 & 4,0 & 14,0 & 1,172 & 0,36 \\
F9 & 16,5 & 6,0 & 21,0 & 9,0 & 14,0 & 1,181 & 0,13 \\
F10 & 14,0 & 6,0 & 13,0 & 5,0 & 12,0 & 0,876 & 0,11 \\
F11 & 19,0 & 8,0 & 22,5 & 1,75 & 14,0 & 1,157 & 0,10 \\
F12 & 15,8 & 10,0 & 11,5 & 8,0 & 12,3 & 1,378 & 0,09 \\
F13 & 18,0 & 6,0 & 24,0 & 10,5 & 14,8 & 1,194 & 0,15 \\
F14 & 17,0 & 8,0 & 18,0 & 6,5 & 13,0 & 1,038 & 0,12 \\
F15 & 19,3 & 8,5 & 21,5 & 9,0 & 12,2 & 1,184 & 0,14 \\
F16 & 19,5 & 9,5 & 20,5 & 10,0 & 12,3 & 0,886 & 0,12 \\
F6 & 15,0 & 8,5 & 13,0 & 11,0 & 12,0 & 1,470 & 0,39 \\
F17 & 13,5 & 7,0 & 13,0 & 6,5 & 12,5 & 1,053 & 0,07 \\
F18 & 15,3 & 8,0 & 14,5 & 12,0 & 10,2 & 0,868 & 0,06 \\
F19 & 15,8 & 10,0 & 11,5 & 8,5 & 12,0 & 1,417 & 0,11 \\
F20 & 19,0 & 6,5 & 24,0 & 4,0 & 12,3 & 0,914 & 0,10 \\
F21 & 15,3 & 9,5 & 11,5 & 27,0 & 13,3 & 1,153 & 0,10 \\
F22 & 15,0 & 8,0 & 12,0 & 26,0 & 11,4 & 0,927 & 0,11 \\
F23 & 18,5 & 10,5 & 16,0 & 26,0 & 9,2 & 1,324 & 0,11 \\
F24 & 20,0 & 10,0 & 20,0 & 25,0 & 10,2 & 1,264 & 0,10 \\
F7 & 26,0 & 12,0 & 28,0 & 1,0 & 16,4 & 1,638 & 0,31 \\
F25 & 23,8 & 12,0 & 23,5 & 0,0 & 14,8 & 1,160 & 0,10 \\
F26 & 22,3 & 13,5 & 17,5 & 1,0 & 14,9 & 0,892 & 0,10 \\
F27 & 20,8 & 12,5 & 16,5 & 2,5 & 13,9 & 1,178 & 0,08 \\
F28 & 20,5 & 8,5 & 21,0 & 0,5 & 14,1 & 0,932 & 0,08 \\
F29 & 23,8 & 12,0 & 23,5 & 2,5 & 14,8 & 1,160 & 0,10 \\
F30 & 22,3 & 13,5 & 17,5 & 1,5 & 14,9 & 0,893 & 0,10 \\
F31 & 27,0 & 15,0 & 27,0 & 5,5 & 14,7 & 1,292 & 0,08 \\
F32 & 24,3 & 14,0 & 20,5 & 3,0 & 13,0 & 1,249 & 0,09 \\
Especificações & & & & & & & \\
Petrobrás $[11]$ & $\geq 15,0$ & $\geq 4,0$ & $\leq 1,5 x \mathrm{VP}$ & $\mathrm{NE}$ & $\leq 18,0$ & $\mathrm{NE}$ & $\mathrm{NE}$ \\
\hline & & & & & & & \\
\hline
\end{tabular}

contendo goma xantana, amido, $\mathrm{MgO}, \mathrm{CaCO}_{3}$ e lubrificante (foram avaliadas 10 amostras de lubrificantes) [17]. Os dados apresentados neste trabalho estão de acordo com os da literatura e, para todos os fluidos desenvolvidos, os resultados de CL foram inferiores, confirmando assim a capacidade de lubrificação de fluidos de perfuração à base de água doce, contendo bentonita e polímero. Os excelentes resultados de lubricidade são justificados pelo tipo de aditivo utilizado. $\mathrm{O}$ Lub1 é um lubrificante à base de óleo vegetal, solúvel em água e possui caráter tensoativo, assim, ele forma um filme sobre as superfícies capaz de interações mais fortes que os óleos comuns e proporciona menores e melhores valores de CL; o Lub2 é um éster ácido de cadeia curta e etanol, insolúvel em água e não possui cargas, apenas dipolos e os lubrificantes Lub3 e Lub4 são misturas de óleos vegetais que, por sua vez, são gorduras extraídas de plantas. São ésteres insolúveis em água e formam gotículas que se dispersam no meio líquido. Essas gotículas são extremamente pequenas, dando a impressão de que se trata de um produto solúvel, e são responsáveis pela lubricidade dos fluidos.

É importante ressaltar que os lubrificantes avaliados neste trabalho são ambientalmente corretos e biodegradáveis, apresentando enormes vantagens nos aspectos ambientais, sem agressões ao meio ambiente. E por serem compostos de óleos vegetais, considerados, por sua vez, fontes renováveis de energia, apresentam vantagens sociais e econômicas.

\section{CONCLUSÕES}

Com o objetivo de desenvolver fluidos aquosos para perfuração de poços onshore conclui-se que os fluidos hidroargilosos apresentaram comportamento de fluidos binghamianos e quando aditivados com polímeros e lubrificantes, comportamento pseudoplástico. Ficou evidenciado que os aditivos poliméricos e lubrificantes utilizados no desenvolvimento dos fluidos de perfuração desempenharam com êxito suas funções de modificadores reológicos, redutores de filtrado e agente lubrificante, bem 
como que há a necessidade de aditivos lubrificantes em fluidos aquosos contendo argila bentonítica e polímeros. Por fim, a aditivação com apenas 1,0\% de lubrificante é suficiente para adequar a capacidade lubrificante dos fluidos desenvolvidos.

\section{AGRADECIMENTOS}

À Agência Nacional do Petróleo - ANP, ao PRH-25, à FINEP, ao CTBRASIL, ao CTPETRO, ao CNPq (Proc. 010592/2006-2) pelo apoio financeiro, à Bentonit União Nordeste Ltda. pelo fornecimento da argila bentonítica, à System Mud Ind. Com. Ltda. pelo fornecimento dos lubrificantes e polímeros e ao LABDES pelo uso de suas instalações físicas e apóio à pesquisa.

\section{REFERÊNCIAS}

[1] L. V. Amorim, Melhoria, proteção e recuperação da reologia de fluidos hidroargilosos para uso na perfuração de poços de petróleo, Tese Dr., CCT, UFCG, Campina Grande, PB (2003).

[2] H. C. H. Darley, G. R. Gray, Composition and properties of drilling and completion fluids, $5^{\text {th }}$ Ed., Gulf Publ. Co., Houston, TX, EUA (1988).

[3] J. E. Thomas, Fundamentos de engenharia de petróleo, Ed. Interciência, Rio de Janeiro, RJ (2001).

[4] J. L. Lummus, J. J. Azar, Drillings fluids optimization a pratical field approach, PennWell Publ. Co., Tulsa, OK, EUA (1986).
[5] R. C. A. Medeiros, L. V. Amorim, L. N. L. Santana, Anais XVII Cong. Bras. Eng. Quim., Recife, PE (2008).

[6] M. I. R. Barbosa, L. V. Amorim, H. C. Ferreira, Cerâmica 53, 328 (2007) 354.

[7] L. V. Amorim, M. S. Pereira, H. C. Ferreira, Anais Rio Oil \& Gas Expo and Conf., Rio de Janeiro, RJ (2008).

[8] A. Dzlalowski, S. Mahajan, paper SPE/IADC 25730 (1993) 501.

[9] H. O. Gomes, J. F. O. Filho, Encontro para a Qualidade de Laboratórios, S. Paulo, SP (2005).

[10] Petrobrás - Argila aditivada para fluido de perfuração à base de água na exploração e produção de petróleo, Especificação N-2604 (1998).

[11] Petrobrás - Argila aditivada para fluido de perfuração à base de água na exploração e produção de petróleo, Método, N-2605 (1998).

[12] K. V. Farias, L. V. Amorim, H. C. Ferreira, E. Pereira, Cerâmica 52, 324 (2006) 307.

[13] API, Norma API Recommended practice 13B-1 (2003).

[14] L. V. Amorim, C. M. Gomes, F. L. H. Silva, H. L. Lira, H. C. Ferreira, Águas Subterrâneas 19, 1 (2005) 75.

[15] R. C. A. Medeiros, L. V. Amorim, L. N. L. Santana, Estudo do coeficiente de lubricidade de fluidos de perfuração à base de água, Monografia (PRH-25/ANP), CCT, UFCG, Campina Grande, PB (2009).

[16] F. B. Growcock, L. A. Sinor, A. R. Reece, J. R. Powers, paper SPE 28708 (1994) 393.

[17] D. Knox, P. Jiang, paper SPE/IADC 92002 (2005).

(Rec. 27/05/2009, Rev. 27/11/2009, Ac. 28/11/2009) 